\title{
Differentiated Services Based Admission Control and Multi Path Routing Algorithm for IPv6
}

\author{
Muhammad Omer Farooq* and Sadia Aziz*
}

\begin{abstract}
In this paper we propose a Differentiated Services Based Admission Control and Routing Algorithm for IPv6 (ACMRA). The basic DiffServ architecture lacks an admission control mechanism, the injection of more QoS sensitive traffic into the network can cause congestion at the core of the network. Our Differentiated Services Based Admission Control and Routing Algorithm for IPv6 combines the admission control phase with the route finding phase, and our routing protocol has been designed in a way to work alongside DiffServ based networks. The Differentiated Services Based Admission Control and Routing Algorithm for IPv6 constructs label switched paths in order to provide rigorous QoS provisioning. We have conducted extensive simulations to validate the effectiveness and efficiency of our proposed admission control and routing algorithm. Simulation Results show that the Differentiated Services Based Admission Control and Routing Algorithm for IPv6 provides an excellent packet delivery ratio, reduces the control packets' overhead, and makes use of the resources present on multiple paths to the destination network, while almost each admitted flow shows compliance with its Service Level Agreement.
\end{abstract}

Keywords: Differentiated Services (DiffServ), Admission Control, IPv6, QoS Routing, QoS Architecture

\section{Introduction}

During the past few years two architectures have been developed primarily for providing Quality of Service (QoS) in Internet Protocol (IP)-based networks, namely, Integrated Services (IntServ) and Differentiated Services (DiffServ). Multi -Protocol Label Switching (MPLS) has emerged as another traffic engineering architecture. Initially, MPLS was invented to provide a fast switching mechanism i.e. instead of conducting a longest prefix match (a major obstacle to improving the performance of routers), a small label is used to index the routing table, so it is only necessary to swap the incoming label with the outgoing label. Many researchers have tried to exploit the capabilities of MPLS to provide QoS provisioning. We know that IP provides the "best effort service", i.e., the IP tries to deliver data to the intended receiver by trying its best, and the Transport Control Protocol (TCP) ensures reliable, in order delivery of data segments on top of IP. Nevertheless, neither of these protocols - along with the User Datagram Protocol (UDP) provides any bandwidth, delay and jitter guarantees to real time applications. IP, TCP and UDP were developed for elastic applications such as the File Transfer Protocol (FTP), the Hyper Text Transfer Protocol (HTTP), and Electronic Mail (Email): such applications need error

Manuscript received 10 March, 2009; accepted 15 May, 2009.

Corresponding Author: Muhammad Omer Farooq

* P-61 Hammed Plaza Congress St \# 6 Jhang Bazar Faisalabad, Pakistan (omer.farooq@ymail.com, sadia.aziz@case.edu.pk) free and in- order delivery of data therefore, no real requirements regarding guaranteed bandwidth and delay. Real time applications like Voice over IP (VoIP), Video Conferencing, IPTV and online gaming require some sort of guaranteed bandwidth, minimum delay and jitter. Since the basic philosophy behind these technologies is to use a widespread, economical IP network infrastructure, we need to invent some mechanism on top of IP to meet the real time requirements of such applications. The Differentiated Services Based Routing and Admission Control for IPv6 represent a step towards meeting the aforementioned requirements of real time applications. Indeed, the Differentiated Services Based Admission Control and Routing Algorithm for IPv6 provides an admission control mechanism for DiffServ based networks along with a DiffServ based routing protocol. Our admission control mechanism makes sure that the amount of real time traffic remains within the limits of the capacity of the network and also tries to increase the throughput of the network by making use of alternate paths (if present). The Differentiated Services Based Admission Control and Routing Algorithm constructs Label Switched Paths, thereby decreasing the switching time compared to the time required for performing the longest prefix match.

This paper has been organized into the following sections: section 2 presents a review of some important work related to QoS in the Internet; section 3 presents the details of the Quality of Service based Multi-path Routing algorithm; Section 4 presents the Differentiated Services Based 
Admission Control and Multi - Path Routing Algorithm for IPv6; section 5 presents the results of the simulations; and, finally, Section 6 presents the conclusions drawn from this research.

\section{Related Work}

In the past few years, work on the Internet Protocol for providing QoS has resulted in two dominant models, i.e., the Integrated Services (IntServ) [1] and the Differentiated Services (DiffServ) [2] models. IntServ tries to provide hard QoS guarantees on a per flow basis, to which end it uses the signaling protocol known as the Resource Reservation Protocol (RSVP). RSVP uses the IP uni-cast and multi-cast routing algorithms, and maintains the flow information, i.e., flow label, minimum bandwidth, delay, and packet loss on each hop along the path. RSVP stores the state information in a soft manner, i.e., it is necessary to send keep-alive messages into the network periodically in order to keep reservations alive; otherwise, timeout will release the resources. One Major drawback associated with IntServ is its scalability issue. As the number of flows increases, the state information that we need to maintain on the routers along the path also increases, and during the course of this process the memory of the routers/switches may be exhausted. Secondly, since RSVP uses IP routing to reserve resources along the path, all the traffic to the same network will reserve the resources along a single path and the admission control of IntServ will deny admission to flows that are destined to the same network when no more free resources are available along a path. In most cases, there exist multiple paths to the same network, so if we deploy a mechanism to dynamically discover these paths and then send traffic on the appropriate path, more traffic can be injected into the network, thereby increasing the throughput of the overall system and ensuring better QoS for the applications.

DiffServ [2] was invented to circumvent the scalability problem of IntServ by providing QoS provisioning on class based granularity. The DiffServ model has defined three main types of traffic classes: Expedited Forwarding (EF) [4]; Assured Forwarding (AF) [5]; and best effort forwarding. Traffic requiring a guaranteed bandwidth, minimum loss, delay and jitter are mapped to the EF class. The AF class is further divided into four classes, each of which has three levels of drop precedence; different flows are mapped to different categories of the AF classes depending upon their needs. The Best effort class of DiffServ treats the traffic in the same way as IP does. Flows corresponding to different DiffServ classes are marked with a code point known as the Differentiated Services Code Point (DSCP). In IPv4 the
"TOS" byte is used to mark the DSCP of a particular flow. In IPv6 this can be achieved by using the "Traffic Class" field of IPv6 packet header. The Standard DiffServ model does not have any admission control procedure to limit the traffic w.r.t. to the capabilities of the network. This limitation can degrade the performance of already established flows when an excess of QoS sensitive traffic is injected into the network. Although numerous admission control procedures have been introduced for DiffServ in the published literature, these solutions have their own limitations. Secondly, similar to the IntServ model, DiffServ relies on IP routing; therefore, it inherits the same problem as that pointed out for IntServ in a similar context.

IPv4 provides a rudimentary form of QoS by only providing the "TOS" byte in its header. the QoS limitations of IPv4 can be judged by inspecting the header of the Internet Protocol version 6 (IPv6), in which two fields are exclusively reserved for QoS in the IPv6 Header, i.e., the "Traffic class" field and the "Flow Label" filed. The former is primarily used to classify traffic related to various classes, and then schedule each type of traffic according to its priority. This field is typically used by the DiffServ model. The latter is used to identify a particular flow among various other ongoing flows, such as when a field is primarily used by the IntServ style of providing QoS which is based on per flow granularity. We will combine the use of these two fields to come up with a very elegant solution for providing QoS and traffic engineering mechanisms on the Internet.

Multi protocol Label Switching (MPLS) [3], which was initially not intended for providing QoS now provides various traffic engineering features (TE). In fact, it is a switching architecture that can route the flow in the network with enhanced speed compared to the traditional longest prefix match lookup performed by the routers. MPLS builds a label switching table known as the Label Forwarding Information Base (LFIB); thus, whenever a packet arrives that contains a label, the MPLS enabled router will look for the label in its LFIB and swap the incoming label with the outgoing label. The MPLS standard header contains a three bit field for QoS, which means that MPLS can differentiate among eight service classes these eight classes of traffic are not sufficient to support the varying QoS needs for different types of real time applications. The constraint based Routing with the Label Distribution Protocol (CR-LDP) is an enhancement which was introduced to MPLS to perform the traffic engineering functionality. CR-LDP treats QoS in a manner akin to that of IntServ; therefore, it suffers from the scalability problem. For MPLS, a new version of RSVP known as RSVP-TE (TE stands for Traffic Engineer) has been defined, while in RSVP-TE a new object of EXPLICIT-ROUTE has been 
introduced. Using EXPLICT-ROUTE we can pinpoint the route that a flow should traverse in-order to reach its destination. RSVP-TE has two shortcomings in that (1) we need to manually configure the labels on our known path, and that (2) the Problems that were reported for RSVP still exist with RSVP-TE.

In [8], the Admission Control over Assured Forwarding PHB was presented. This scheme suffers from following flaw. If AF2 packets (signaling packets) from two different sources manage to find a path and have at least one common node between them, if that node can only accommodate one more flow since none of the two flows have started therefore; their signaling packets will get through but when both of the sources begin to transmit, congestion can occur, thus effecting the performance of all the admitted flows. In $[9,10]$ admission control schemes were presented for the DiffServ architecture. The admission control scheme presented in [9] suffers from the same problem as that mentioned for [8].

\section{Quality of Service based Multi-path Routing Algorithm}

The Quality of Service based Multi-path Routing Algorithm (QoS-MPR) was presented in [11]. QoS-MPR provides an admission control and multi-path routing algorithm for the DiffServ based network. To provide QoS in IP networks, QoS-MPR uses the notion of the label switched path. Each time we want to start a real time flow, the algorithm proposes to broadcast the route request message according to the required QoS parameters. This route request message is forwarded by a receiving node (if the receiving node is not a recipient of a route request message) to its immediate neighbors. Each forwarding node assigns a locally unique identifier to the route request message in order to discriminate between the various route request messages that a node has broadcast. The route reply is only generated by the destination node, and the route reply message goes to the source node on a reverse path.

\subsection{QoS-MPR Route Request Processing}

In QoS-MPR each node will allocate a certain amount of bandwidth to each DiffServ class depending upon the type of service a particular class offers. Each node will keep track of the current total bandwidth allocation in each DiffServ class. The decision to admit a new flow in a particular DiffServ class depends on the priority of the class in which the flow is needed, the total bandwidth allocation in that particular class, the bandwidth requirement of a flow, the average queue occupancy of a particular class in which a new flow is needed, and the deviation of the average queue length from the current committed bandwidth. Every node will use the following formula to compute the average queue length over a specified period of time ' $t$ ', as given in [11].

$$
\text { Average Queue Length }(\mu)=\frac{\sum_{t=t_{0}}^{t_{1}} \text { Queuelength }{ }_{i, t}}{t_{1}-t_{0}}
$$

In the above equation $\mathrm{t} 1-\mathrm{t} 0$ represents the number of seconds over which we are taking the average queue length.

$$
\begin{gathered}
C_{B}=\sum_{n=1}^{N} \text { FlowsCommittedBandwidth }[n] \\
C_{B}=\text { Committed Bandwidth in a given DiffServ class }
\end{gathered}
$$

\section{Deviation from the committed bandwidth $(D B)=C B-u$ (3)}

Now we need to calculate the possible available bandwidth PB: if the value for this possible bandwidth is greater than the bandwidth required by a flow, then the flow will be admitted; otherwise the flow will be rejected.

$$
P B=(T B-C B)+D B+P L
$$

In the above equation TB represents the total bandwidth allocated to a DiffServ class, PB represents the possible available bandwidth, and PL represents the percentage loss in bits that a particular DiffServ class can afford. The value for PL depends on the characteristics of a particular DiffServ class. The PL value for the Expedited Forwarding class will be set to 0 , as the expedited forwarding class tries to guarantee no packet loss. Similarly, the PL parameter for other classes will be set accordingly.

The Following figure shows the format of the route request message given in [11].

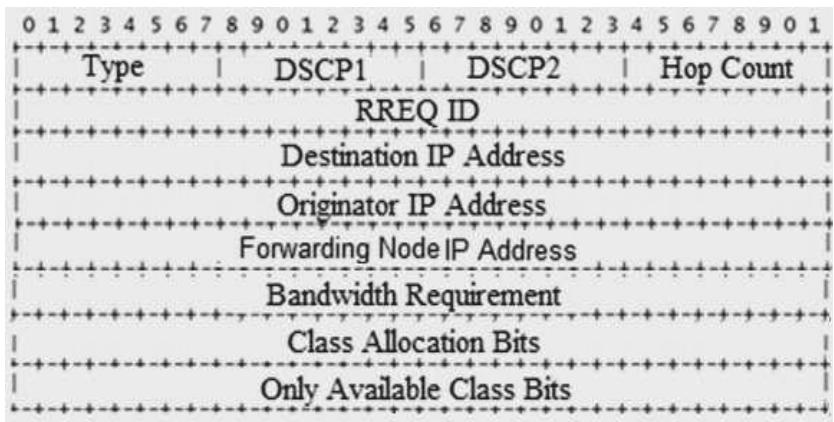

Fig. 1. QoS Route Request Message

\subsection{QoS-MPR Route Reply Processing}

The Following figure shows the format of the route reply message as given in [11]. 


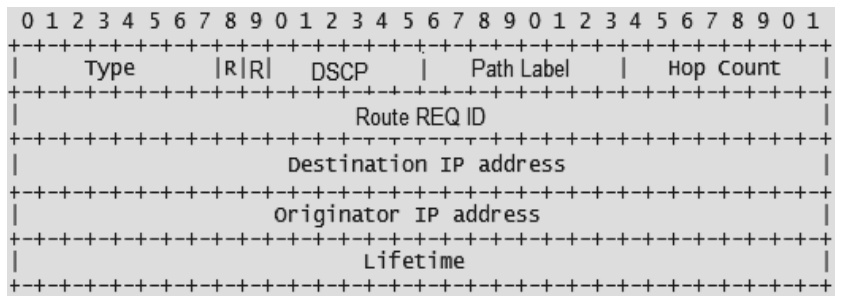

Fig. 2. QoS Route Reply Message

In the above route reply message the type field will have a value of 2 to indicate that this is a route reply message. The next two bits are reserved for future use. The DSCP field will inform the service class selected for this particular flow. The label given in the Path Label field will be used by the upstream node to forward packets belonging to this particular flow to its downstream node. Every downstream node in the path will select a locally unique label for the flow and inform its upstream mode of its selected label using the path label field. The Route REQ ID will contain the Request ID that was mentioned in the route request message; the Route REQ ID along with the originator IP address will enable the receiver of the route reply message to match the reply with the appropriate route request message. The Destination IP address will contain the IP address of the node for which a route was required. The lifetime field will inform the receiver about the interval between two successive packets of the flow. If no packet is received within this interval, the routing table entry pertaining to this particular flow will be deleted.

\subsection{QoS Route Lost Message Processing}

The QoS route lost message has the following format, as shown in [11].

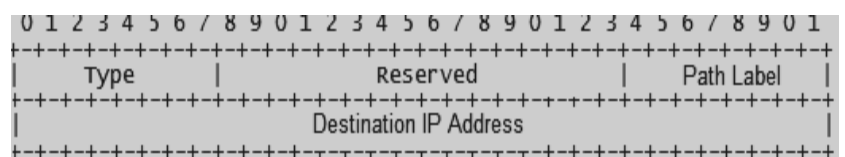

Fig. 3. QoS Route Lost Message

In the case of the QoS Route Lost message, the type field will have the value 3. The path label field in the Qos Route Lost message will have the label that an upstream node is using to send data to this downstream node. Using this Path Label field, every intermediate node will send a QoS route lost message to its upstream node until this message reaches the source node.

\subsection{Shortcomings of QoS-MPR}

In order to comprehend the flaws associated with the QoS-MPR protocol, let us consider the following network topology.

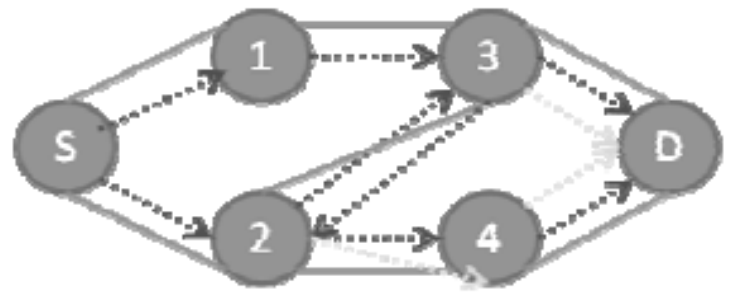

Fig. 4. Network Topology

In the above network the undirected arrows show the bidirectional links between the different nodes present in the network. The red dotted arrows show the propagation of the route request message for the first time. As we can see that there is a loop between the nodes $(2 \rightarrow 3 \rightarrow \mathrm{D} \rightarrow 4$ $\rightarrow 2$ ), the yellow dotted arrows therefore show the propagation of the route request message for the second time.

Let us suppose that node ' $S$ ' has initiated a route request message with the parameters shown in the following table.

Table 1. Flow Specifications by node $\mathrm{S}$

\begin{tabular}{|c|c|c|c|c|}
\hline SRC & DST & DSCP1 & DSCP2 & BW \\
\hline S & D & EF & EF & $4 \mathrm{~KB}$ \\
\hline
\end{tabular}

The source Node 'S' wants to initiate a QoS session with the destination node D. The flow is required in the Expedited Forwarding class, and the bandwidth requirement is 4 kilobytes per second (KB/s).

Let us then assume that the following are the amounts of remaining bandwidth in the selected DiffServ classes at the different nodes present in the network.

Table 2. Remaining Bandwidth at Nodes

\begin{tabular}{|c|c|c|}
\hline Node & EF BW & AF BW \\
\hline S & $80 \mathrm{~KB}$ & $40 \mathrm{~KB}$ \\
\hline 1 & $80 \mathrm{~KB}$ & $80 \mathrm{~KB}$ \\
\hline 2 & $100 \mathrm{~KB}$ & $20 \mathrm{~KB}$ \\
\hline 3 & $80 \mathrm{~KB}$ & $10 \mathrm{~KB}$ \\
\hline 4 & $110 \mathrm{~KB}$ & $70 \mathrm{~KB}$ \\
\hline $\mathrm{D}$ & $0 \mathrm{~KB}$ & $0 \mathrm{~KB}$ \\
\hline
\end{tabular}

The Above table shows that each node can accommodate this flow in the EF class; therefore, each node will reserve $40 \mathrm{~KB}$ of bandwidth during the first propagation of the route request message. Since there is a link between nodes 2 and 3, then according to QoS-MPR, nodes 2, 3 and 4 will rebroadcast this route request message and hence reserve an additional $40 \mathrm{~KB}$ of bandwidth for this flow. Nodes 2, 3 and 4 have reserved $80 \mathrm{~KB}$ of bandwidth for this particular flow due to the second broadcast. This leads to the wastage of bandwidth, which in return may deny admission to future flows. Secondly, equation 4, taken from [11], could 
give us an erroneous estimation of bandwidth. The PL value represents the probability of loss in a given DiffServ class, so once a flow has been admitted using the PL value, that value should be decremented.

\section{Differentiated Services based Admission Control and Multi-path Routing Algorithm for IPV6 (ACMRA)}

The Differentiated Services based Admission Control and Multi-path Routing Algorithm for IPv6 (ACMRA) is primarily an enhancement introduced to the QoS-MPR protocol. ACMRA eliminates the shortcomings of QoSMPR that were listed in section 3.4.

The first problem identified in QoS-MPR concerns over reservation resulting from the duplicate broadcasting of route request messages. We have devised the following mechanism to circumvent this problem.

Whenever a route request message arrives at an intermediate node it will follow the QoS-MPR protocol, apart from the following modification.

Before broadcasting the route request message, the node will check whether it has already reserved a flow in a given range of DiffServ classes; if it has, then the node will not make any additional reservation and will rebroadcast the route request message. If the node has not reserved a flow in the given range of DiffServ classes, then it will make the reservation and rebroadcast the route request message. If

Table 3. Algorithm for Route Request Processing

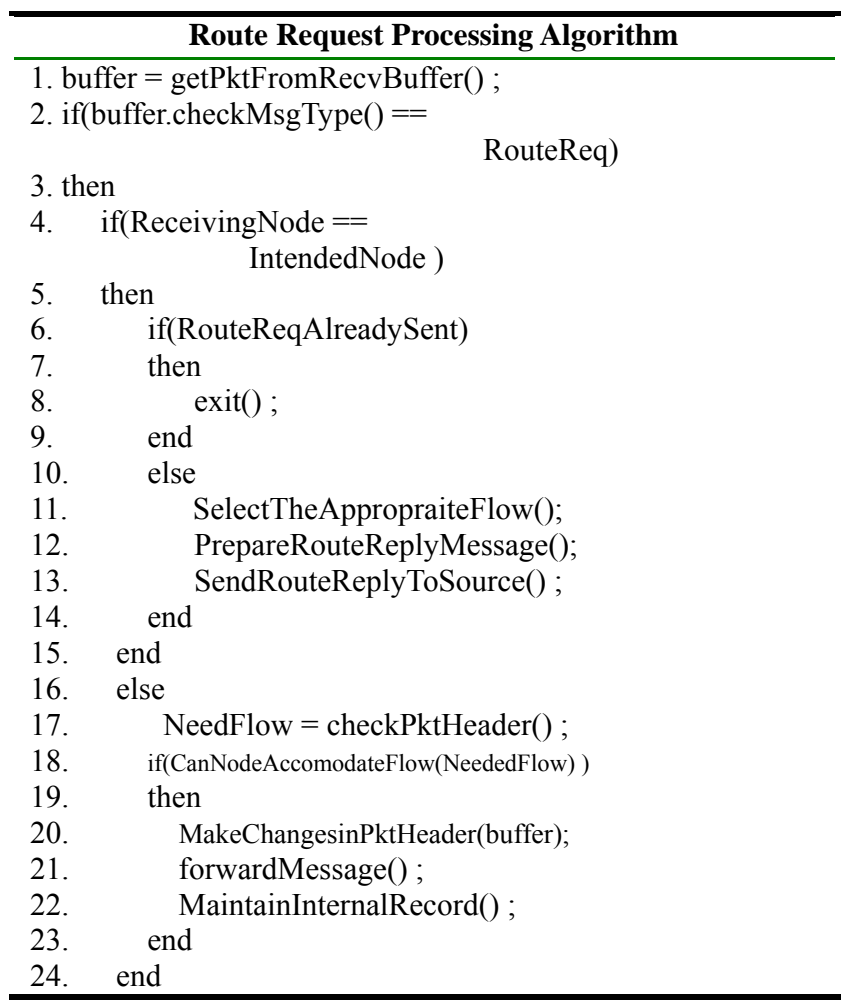

the node has already reserved a flow in a range of DiffServ classes for this route request (i.e., a duplicate route request arriving from a different path) but it has not reserved a flow in a DiffServ class mentioned in this route request, then the node will make the required reservation.

Recalling that in section 3.4 we mentioned that equation 4 can lead to an incorrect estimation of bandwidth, we have devised the following algorithm to estimate accurate bandwidth allocations accurately.

Table 4. Algorithm for Correct Bandwidth Estimation

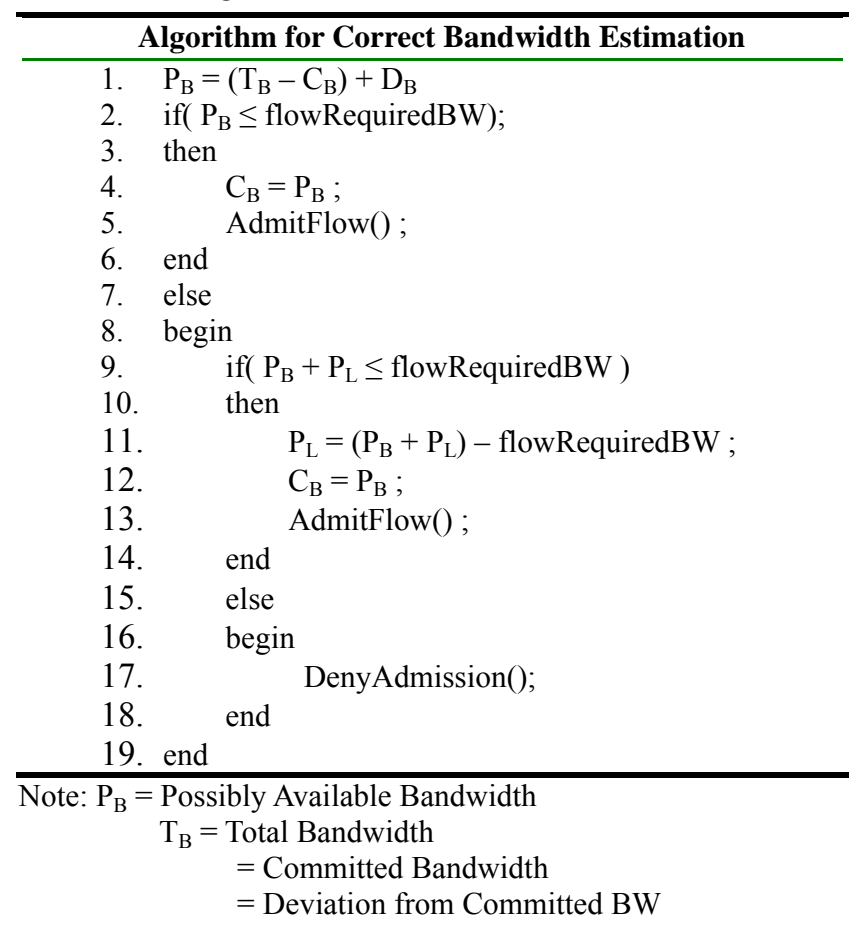

\section{Simulation}

In order to confirm the effectiveness of the Differentiated Services based Admission Control and Routing Algorithm, we compared the performance of the Differentiated Services based Admission Control and Routing Algorithm with QoS-MPR and the standard DiffServ. We performed three sets of simulations, in which we compared the packet delivery ratio, average delay faced by data packets, routing overhead, and the compliance of the admitted flows with their respective service level agreement.

\subsection{Simulation Setup}

We designed a discrete time simulator with the name "Enhanced QoS-MPR" to perform the required simulations. The Following figure shows the graphical interface of the simulator. 


Es Topology Infromation
\begin{tabular}{||c||c||c||}
\hline \multicolumn{1}{|c|}{ Node } & \multicolumn{1}{|c||}{ Links } & bandwidth \\
\hline 0 & 1,3 & 1000000,1000000 \\
\hline 1 & 0,2 & 1000000,1000000 \\
\hline 2 & 1,6 & 1000000,1000000 \\
\hline 3 & 0,4 & 1000000,1000000 \\
\hline 4 & 3,5 & 1000000,1000000 \\
\hline 5 & 4,6 & 1000000,1000000 \\
\hline 6 & 2,5 & 1000000,10000000 \\
\hline \hline & & Update Topology \\
\hline
\end{tabular}

Fig. 5. Topology Information GUI

The Following GUI shows the simulation statistics at the end of the simulation.

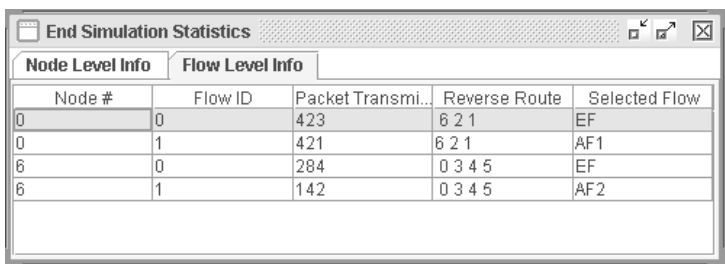

Fig. 6. Simulation Statistics GUI

Each of our traffic sources was mapped to the Expedited Forwarding class. UDP was used as the transport layer protocol and Constant Bit Rate (CBR) traffic was generated. Each data packet consisted of 512 bytes and the interval between the two consecutive data packets was configured to be 0.008 seconds. Hence, the bandwidth requirement for each flow was 500 Kilobits per second. Each link in the network had a bandwidth of 2 megabits per second.

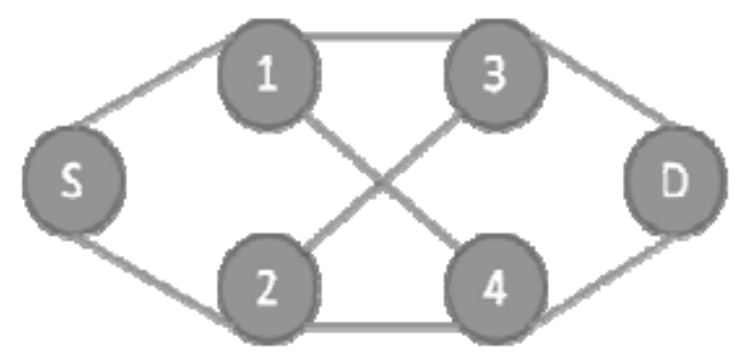

Fig. 7. Simulation Topology

\subsection{Simulation Results}

From Fig. 8 it is evident that the packet delivery ratios of the DiffServ based QoS-MPR and the Differentiated Services Based Admission Control and Multi-path Routing Algorithm for IPv6 (ACMRA) are far better compared to the performance of DiffServ using the traditional link state routing. We know that standard routing algorithms maintain only one route to the particular destination; therefore, all the traffic to that network will be routed through the selected optimal path. When the offered load on the link increases, the packet delivery ratio will decrease; as such, in these types of scenarios it is difficult to meet the QoS requirements of a flow. QoS-MPR and ACMRA try to find multiple paths to the destination and then route the traffic of a particular flow on a path that best suits the requirements of a specific flow. Therefore, if multiple paths exist to the destination, ACMRA and QoS-MPR exhibit much better performance.

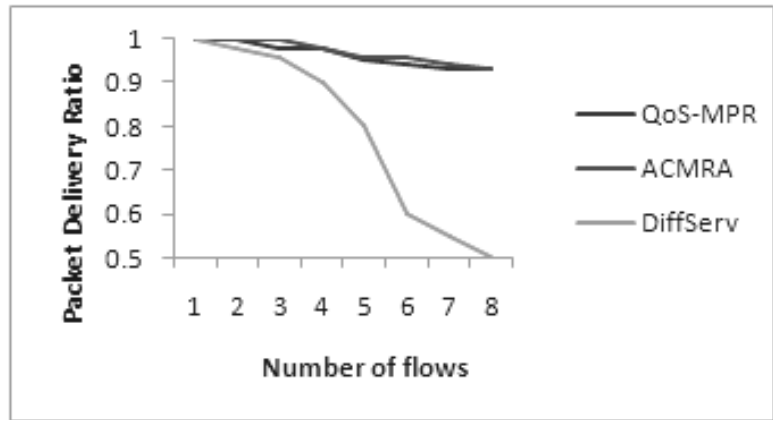

Fig. 8. Packet Delivery Ratio

Fig. 9 shows the plotting of the delay experienced by the Expedited Forwarding (EF) class traffic using link state routing with DiffServ, QoS-MPR and ACMRA. When we simulated DiffServ using Link State routing, an increase in single flow caused the traffic in the network to experience long delays, particularly when the resources on a given link were exhausted by previously established flows. This is a point where we potentially need to direct further flows on a different path if one exists in the network topology, and if no other path exists then we should deny admission to those flows whose QoS requirements cannot be fulfilled. Since the standard DiffServ architecture and traditional network routing algorithms do not provide these functionalities, the delay experienced by all the admitted flows will abruptly increase once the resources on a given link are fully utilized. Conversely, ACMRA and QoS-MPR find multiple paths to the destination and direct flows on to discovered multiple paths, and will not grant admission to those flows whose QoS requirements cannot be fulfilled; hence, far fewer delays are experienced in the case of ACMRA and QoS-MPR, as depicted in the graph.

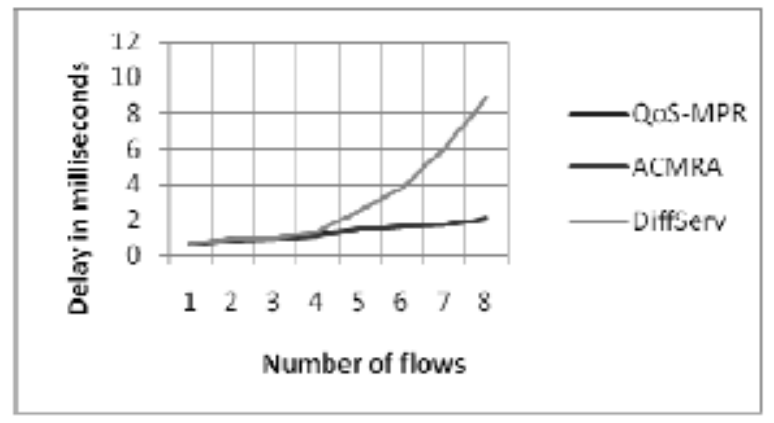

Fig. 9. Delay Comparison 
Fig. 10 show the numbers of reservations versus the number of admitted flows. Since standard DiffServ does not make any reservations within the DiffServ classes, the number of reservations in this case thus remains 0 . In our simulated network topology there are loops; therefore, QoS-MPR makes reservations for the same route request multiple times; hence, the number of reservations in this case is higher than needed. Conversely, ACMRA only makes reservations where necessary, thereby limiting the number of reservations.

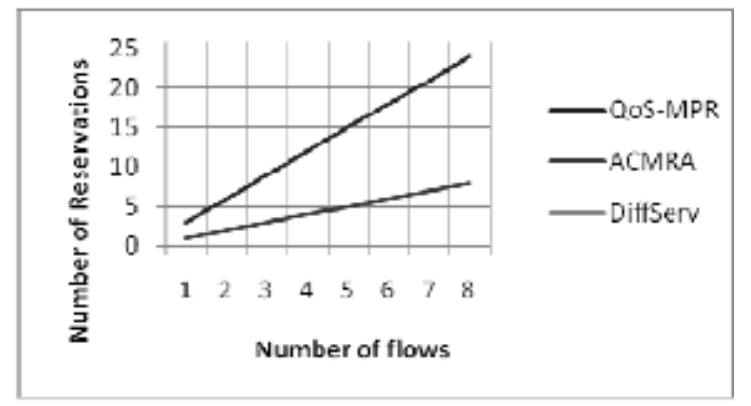

Fig. 10. No. of Reservation Vs. No. of Flows

Fig. 11 shows a graph of the "Number of Flows vs. Compliance with Service Level Agreement SLA". This graph is plotted by incorporating an admission control mechanism into QoS-MPR and ACMRA. We can observe that in the simulated network topology, ACMRA and QoSMPR have restricted the number of EF flows to 8, and that each admitted flow complies approximately $100 \%$ with its SLA. As DiffServ with Link state routing does not employ any distributed admission control mechanism, when the bandwidth requirements exceeds a certain threshold level (Path's bandwidth) there is an abrupt degradation in the quality of the admitted flows. Since SLA corresponding to EF requires no packet loss, the flow's percentage compliance with SLA almost reaches 0 when the bandwidth requirement of all the flows exceeds a certain level.

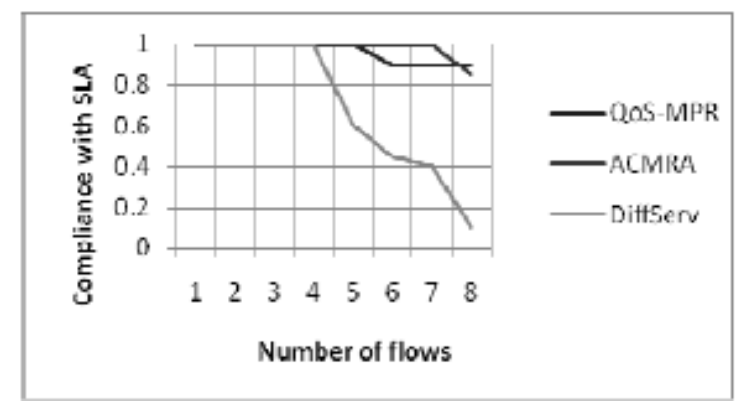

Fig. 11. Compliance with SLA vs. No. of Flows

The Fig. 12 shows a comparison of the normalized routing load of ACMRA, QoS-MPR and DiffServ based traditional routing. The Normalized routing loads of ACMRA and QoS-
MPR are approximately the same as those of traditional routing algorithms when only a few QoS flows are present in the network. With an increase in the number of QoS enabled flows, the routing overheads of ACMRA and QoSMPR exceed the overheads recorded for traditional routing protocols.

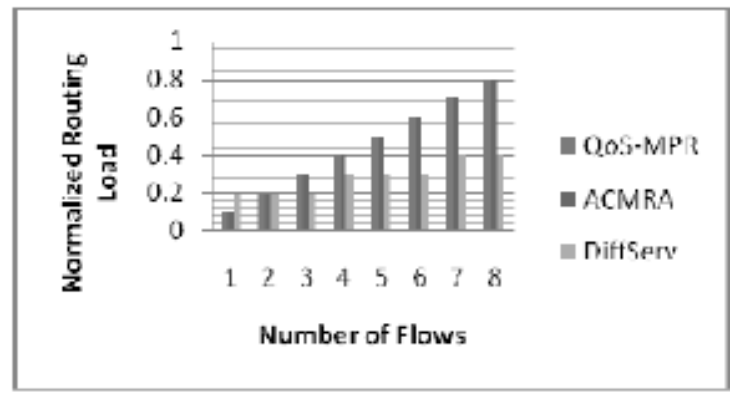

Fig. 12. Normalized Routing Load

\section{Conclusion}

In this paper we have presented the DiffServ based Admission Control and Multi-path Routing Algorithm (ACMRA). ACMRA is primarily an enhancement presented in a previous work on QoS known as QoS-MPR. ACMRA not only tries to find multiple paths that satisfy the QoS requirements of flows, but also provides an admission control mechanism for a DiffServ -based network. ACMRA provides a mechanism that enables the depiction of correct bandwidth allocations within each DiffServ class, as well as making fewer reservations compared to QoS-MPR. The results of the simulation show that ACMRA meets the QoS requirements of all the admitted flows in an effective and efficient manner. Furthermore, it could help to limit the traffic into the network in order that the QoS of already admitted flows remains intact.

\section{References}

[1] R.Barden, D.Clark, S.Shenker, "IntServ in the Internet Architecture an Overview" RFC 1633, June, 1994.

[2] K. Chan, J. babiarz, F. Baker "Aggregation of DiffServ Service Classes", RFC 5127, Feburary, 2007.

[3] E. Rosen, A. Viswanathan, R. Callon "Multiprotocol Label Switching Architecture" RFC 3031, January, 2001.

[4] J. Bennet, Kent Benson, Angela Chiu, Sharama Davari, C.Kalmanek, D.Stiliadis, B.Davie, A. Charny, F.Baker, J. L.Boudec, W. Courtney, V. Firioiu K.K. Rama-krishnam, "An Expedited Forwarding PHB", RFC2598, March, 2002. 
[5] J. Heinanen, f. Baker, W. Weiss, J.Wroclawski, "Assured Forwarding PHB Group", RFC 2597, June, 1999.

[6] B. Jamoussi, R. Callon, R. Dandu, L. Wu, P. Doolan, N. Feldman, T. Worster, A. Fredette, M. Girish, E. Gray, J. Heinanen, T. Kilty, A. Malis "Constraint based LSP set using LDP", RFC 3212, January, 2002.

[7] D. Awduche, L. Berger, D. Gan, T. Li, V.Srinivasan, G. Swallow, RSVP-TE: Extensions to RSVP for LSP Tunnels", RFC 3209, December, 2001.

[8] G. Bianchi, N. Blefari-Melazzi, "Admission Control over Assured Forwarding PHBs: a way to provide Service Accuracy in a DiffServ Framework", Global Telecommunications Conference San Antonio 29, Nov., 2001, Vol.4, pp.2561-2565.

[9] M. Li, B. Hoang, A. Simmonds, "Fair Intelligent Admission Control over DiffServ Network", 11 IEEE International Conference on Networks, pp.501-506, Oct., 2003.

[10] E. Mykoniati, C. Charalampos, P. Geogatsos, T. Damilatis, Algonet, D. Goderis, P. Trimintzios, G. Pavlou, "Admission Control for Providing QoS In DiffServ IPNetworks: The TEQUILA Approach", IEEECommunication Magzine, Vol.4 pp.38-44, Jan., 2003.

[11] Muhammad Omer Farooq, Sadia Aziz, "QoS based Multipath Routing Algorithm for IPv6, 12th IEEE INMIC, 23-24, December, 2008, Karachi, Pakistan.

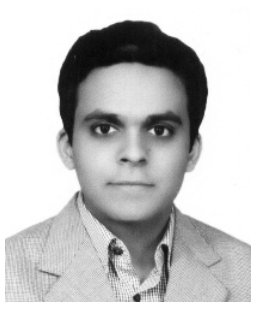

\section{Muhammad Omer Farooq}

Muhammad Omer Farooq has received his BS (Computer Science) and MS (Computer Engineering) degrees with distinction from Virtual University of Pakistan and Center for Advance Studies in Engineering Islamabad, Pakistan respectively. He is a $\mathrm{PhD}$ student at Ottawa Carleton Institute of Electrical and Computer Engineering. His research interest lies broadly in computer and wireless mobile networks, in particular he is interested in research issues related to MAC and upper layers of wireless mobile and sensor networks.

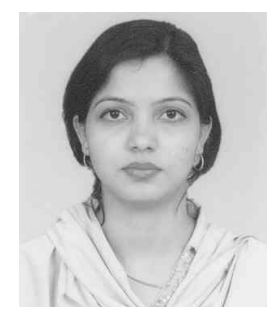

\section{Dr. Sadia Aziz}

Dr. Sadia Aziz is Assistant Professor at Center for Advance Studies in Engineering, Islamabad, Pakistan. She has received her $\mathrm{PhD}$ in Computer Engineering from Wuhan University of Technology China. Her research interest lies in cross layer design of multicast and broadcast algorithms for Mobile Ad hoc Networks. She has also done extensive research related to Quality of Service (QoS) issues in MANET. 